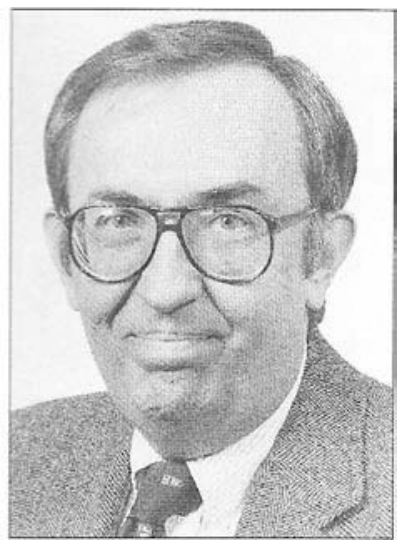

FREDERICK A. MURPHY
Dean, UC Davis School of Veterinary Medicine

\title{
Veterinary Medicine in Service to Animal Agriculture
}

In this era of amazing technological advances and rapidly changing international economic systems, America's continued success in animal agriculture depends upon healthy animals and the assurance that food products are safe and of high quality. Livestock in America today are the healthiest in history, and the foods they provide to us are the safest and best ever. Yet, the public wants and expects more.

Veterinary medicine, as the most diverse and all-encompassing health science, provides comprehensive leadership which helps to maintain a plentiful supply of safe and wholesome foods of animal origin. This service to society reflects the profession's commitment to animal agriculture. Increasing numbers of veterinarians are pursuing advanced training in livestock production, health management, and related fields which enhance animal productivity and well being. Their Hippocratic commitment to heal combined with objective, science-based knowledge equips veterinarians to advance animal agriculture in partnership with diverse groups of professionals including livestock producers, animal scientists, extension specialists, food processors and distributors, and government advisory and regulatory agencies. There is great opportunity, but there are also great challenges.

If animal health is one of the cornerstones of our animal production economy, then how will veterinary medicine maximize livestock health and minimize the impact of disease? One answer lies in expanding the concept of herd health management to include the principles of preventive medicine. Borrowing some terms from the human-health sector, today's animal health/preventive medicine framework can be divided into three major themes:

- Disease prevention strategies. This is the traditional model for preventive services. It is based on early detection, screening, and treatment, and relies in part on one-on-one veterinarian-patient interaction. These interventions generally take place within traditional veterinary-care delivery systems. Wider-ranging interventions set in the public sector include some of the most successful health strategies ever devised, such as vaccine development, disease surveillance, and vector control. These strategies greatly enhance the delivery systems for veterinary medicine.

- Health promotion strategies. The human-health model uses a broad array of strategies such as exercise, smoking cessation, and healthful diets to encourage improved health. These behavioral changes require a modification of the individual's knowledge and attitudes. By analogy, veterinarians who practice herd health management will spend more time with farm- ers than with their cows, but the results will have a substantial health dividend.

- Environmental prevention strategies. In the human-health sector, environmental strategies (health protection strategies) such as safe water, fluoridation, lead abatement, regulating public smoking, seat belt laws, and safer highways generally need a major societal commitment before necessary interventions can take place. Once these interventions are set, they require little individual effort on the part of those who benefit, and can have far-reaching implications. We are just entering this arena in livestock agriculture, and the role of the veterinarian as the "doctor for the environment" is about to be explored.

As is the case in the human-health sector, would it not be in the best interest of producers and others concerned with animal agriculture to consolidate these elements into a single approach? I believe the answer is yes, but this will require great wisdom and some overriding sense of what is good for all producers and all citizens of our country.

In this regard, the dark side of the cloud is represented by the anti-science forces, who are involved in worldwide debates over biotechnology and animal well-being. Critics argue that supplies of meat and milk are more than adequate and question the reasons for conducting biotechnological research, which, in their opinion, only results in larger surpluses. They say that biotechnologically derived products are unproven and inherently dangerous. If science is allowed to generate improved and more efficient production practices, critics further contend, this will only cause additional economic imbalances.

To address societal concerns such as these, veterinary medicine is entering the world of public education and public information in a very substantial way. Veterinary medicine is also forming comprehensive partnerships with livestock specialists and producers to develop and apply the necessary technology that maintains the vitality and improves the productivity of the foodproducing livestock industries. At the same time the profession will continue to supply the expertise that ensures the safety and wholesomeness of foods of animal origin.

Our country's agricultural prosperity lies in quality management programs that maximize production health, that assure animal well-being, that improve the quality of the product leaving the farm gate, and that provide environmentally sound production systems. Veterinary medicine stands ready to redefine its role as an agent for change to meet the needs of the consumer, the producer, and society. 